\title{
Atom-Diatom Collision Processes: Rovibrationally Detailed Cross Sections For Models
}

\author{
Fabrizio Esposito \\ Istituto di Metodologie Inorganiche e Plasmi (IMIP), Consiglio Nazionale delle Ricerche \\ via Orabona 122/D, 70126 Bari (Italy)
}

\begin{abstract}
Atom-diatomic molecule collision processes are of particular importance in nonequilibrium numerical models in which rovibrational energy exchange and state-selected dissociation are taken into account by means of rate coefficients. If also translational nonequilibrium is considered, availability of large sets of cross sections is needed. To cope with this issue extended quasiclassical calculations have been performed to obtain translational energy dependent detailed data for hydrogen, nitrogen and oxygen, with particular attention devoted to computational optimization. Problems related to huge memory requirements of large cross section sets when used in numerical models can be effectively solved with an interpolation method proposed by the author, which seems to reach an optimal compromise between accuracy and amount of data storage required. Comparisons with literature are, when available, globally good. An important issue about the exclusion of rotational distribution in both vibrational energy exchange and dissociation rate coefficients is stressed. Rotational distribution can significantly change dynamical results, with obvious consequences in their applications to models, independently of its explicit or implicit consideration into models. Concerning collision induced dissociation/recombination dynamics, a strong rotational dependence is shown using two different mechanisms in the case of hydrogen.
\end{abstract}

Keywords: collision process, atom diatom cross section, vibrational energy, dissociation, recombination PACS: $\underline{34.50 . \mathrm{Ez}, 1.70 . \mathrm{Hq} 3,82.20 . \mathrm{Bc}, 82.20 . \mathrm{Rp}}$

\section{INTRODUCTION}

Vibrational nonequilibrium in numerical models treating collisions of molecules is recognized as one of the most important aspects to take into account for obtaining accurate and reliable results [1-5]. This means, among other requirements, the need for vibrationally detailed rate coefficients for atom-diatom collision processes. Typical relaxation time for vibration is significantly higher than for rotation and translation of molecules, therefore it is possible to deal with a "vibrational kinetics", while equilibrium can be assumed for rotational and translational degrees of freedom. When this is the case, however, vibrational rate coefficients should take into account the appropriate translational and rotational conditions simulated by the model during its time evolution $[4,5]$. There are two kinds of approximation that are generally performed with this respect: the first consists of treating rotation and translation as in equilibrium at a given temperature, while the second consists of simply neglecting any explicit dependence on rotation, approximation generally coming from the details of the dynamical method used for calculating rate coefficients. In this last case it can be quite uncorrect to neglect the effect of rotation, as will be shown below, in particular for dissociation/recombination kinetics, but also for vibrational energy exchange. When also translational nonequilibrium must be taken into account, for example by means of direct Monte Carlo methods, an explicit dependence on translational energy is required, with much more detailed data (cross sections). Also in this case rotation can be neglected, or considered with a Boltzmann distribution at a given rotational temperature, or explicitly taken into account with detailed cross sections, depending at least on initial rotation, with summed up or explicit final rotation. However this level of detail becomes rapidly a problem even for a small number of chemical species involved into model, not simply for storage, but for management of data along the kinetic simulation. For the hydrogen molecule, for example, there are about 350 rovibrational states, but possible transitions among these states are of the order of $350^{2}$. For other species, as nitrogen or oxygen, the situation is even worse, with about 10000 and 6000 states respectively to consider. Therefore it is fundamental to study methods to keep as low as possible the amount of data to manage, once those data are available for models. In the past ten years a significant effort has been done in order to assemble a database of cross sections for the collision process: 


$$
A+A^{\prime} A^{\prime \prime}(v, j) \rightarrow\left\{\begin{array}{cc}
A+A^{\prime} A^{\prime \prime}(w, k) & \text { non-reactive } \\
A A^{\prime}(w, k)+A^{\prime \prime}, A A^{\prime \prime}(w, k)+A^{\prime} & \text { reactive (atom exchange) } \\
A+A^{\prime}+A^{\prime \prime} & \text { dissociation }
\end{array}\right.
$$

Here $\mathrm{v}, \mathrm{w}$ are respectively initial and final vibrational quantum numbers, while $\mathrm{j}, \mathrm{k}$ are initial and final rotational quantum numbers of the diatom. A, A', A" are atoms of only one species (hydrogen [6-7] or nitrogen [8-11] or oxygen [12-14], a general review in [15-16]). Cross sections have been obtained in a homogeneous way with only one dynamical method, the quasiclassical one (QCT), which is a good compromise between global accuracy and computational resources required. In the next paragraph some details of the calculations will be presented, with related problems and solutions adopted. The successive paragraph is dedicated to a novel technique proposed in order to cope with the problem of managing huge amounts of detailed quasiclassical cross sections into kinetic models, by representing these data in a particular way which preserves accuracy with an extremely low number of data in comparison to the level of detail. The fourth paragraph deals with some comparisons with literature results, in order to assess the validity of the database. In the fifth paragraph general trend of rotational rate coefficients in a specific vibrational transition are studied. Recombination rate coefficients for hydrogen are presented in the sixth paragraph to underline their relevant dependence on rotation. Conclusions are presented in the last paragraph.

\section{CROSS SECTIONS}

\section{Cross section calculations}

Detailed calculations have been performed using a set of numerical codes developed by the author for this purpose initially in a simple serial version during the preparation of his $\mathrm{PhD}$ thesis [17], and then improved for including the possibility of parallel and distributed calculations, with a semiautomatic procedure with respect to the completion of rovibrational ladder as a set of initial states. Quasiclassical method is particularly suited for distributed computations, and this has been deeply exploited by using grid computing, with the aid of some additional procedures for checking the results obtained and eventually discard them, given the high probability of failure typical of this kind of environment. Particular attention has been devoted in the course of these years to the optimization of the ratio between accuracy and computational time step. At each time step, the integration of classical equation of motion of the collisional system in the forward direction is compared with a very small tolerance on both positions $\left(10^{-9} \AA\right)$ and velocities $\left(10^{-9} \AA / \mathrm{fs}\right)$ with a reverse integration: if the test fails, the time step is decreased (halved) and the procedure repeated, until a maximum of 5 or 6 times in case of failures. Starting with a time step empirically good for $90-95 \%$ of trajectories, it is possible to reach a good compromise between accuracy and computational time, because very often the initial time step is conserved, while only rarely very complex or critical trajectories require much smaller time steps. On the other side, these complicated trajectories are treated accurately, with a fine time step, and anyway each trajectory is checked, contrarily to the common use of one check in ten, which has only a statistical sense but do not avoid inclusion of wrong trajectories into the results. In this case of large sets of cross section calculations it is necessary to guarantee accuracy of each trajectory, because some low probability results depend critically on very few integrations [12].

Concerning collision process involving hydrogen: $\mathrm{H}+\mathrm{H}_{2}(\mathrm{v}, \mathrm{j})$, the potential energy surface (PES) used in all the calculations has been the BKMP2 [18], which is known as one of the best PES for this system, with accurate description of interaction also for high lying rovibrational states. In these calculations also the quasibound states have been considered as initial and possible final states, leaving to the kinetic simulation the possibility of including transitions to quasibound states into dissociation or not, depending on the specific conditions. Transitions from quasibound to bound states are of particular importance in the theory of recombination, as shown below. For the hydrogen case the cross section database is complete, in the sense that all the possible rovibrational energy exchange and dissociation transitions have been calculated, with translational energy from $1 \mathrm{meV}$ to $9 \mathrm{eV}$. Stratified sampling has been applied, maximum interaction distance is fixed to $12 \AA$ and energy axis has been scanned in a continuous way. Density of trajectories is at least 24000 per $\AA$ of impact parameter and per eV of translational energy.

Concerning nitrogen and oxygen the huge number of states has dictated a different approach: as initial states some vibrational states have been chosen with all the related rotational states, including all the vibrational ladder for final states, with a summed (not distinct) final rotation. It is not prohibitive to think of extension of database to cover to entire initial vibrational ladder, but applications in models of these data up to this moment have not required such 
level of detail. In fact, only rate coefficients have been necessary, with suited interpolation formulas to cover the lacking initial vibrational states $[11,14]$. It should be noted that interpolation of rate coefficients on initial vibration, temperature and quantum jump is relatively easy, anyway much simpler than the analogous operation on cross sections. The next paragraph will present the interpolation method proposed for cross sections. Concerning nitrogen, the PES adopted is the well known LEPS of Lagana' et al. [19], which is a semiempirical surface. At the time of calculations no other PES was available, while now there are different examples of more refined surfaces [20,21], in particular the PES of Varandas and collaborators [22], on which new sistematic calculations will be soon performed. The calculations in the database have a minimum density of 24000 trajectories per $\AA$ of impact parameter and per eV of translational energy in the interval 0.001 to $3 \mathrm{eV}$, and 4 times less for higher energy up to $10 \mathrm{eV}$. Other conditions are analogous to those for hydrogen, but maximum interaction distance, which is $15 \AA$. Initial vibrational states are: $0,1,3,5,7,10$, then 1 in ten up to 60, then 65, 66 and 67 (the PES adopted supports 68 vibrational levels with a maximum of 261 rotational levels for $\mathrm{v}=0$ ).

Concerning oxygen, the DMBE PES of Varandas and Pais [23] has been used. Even if there are more recent accurate PESs proposed now for this system [24,25], comparisons with experimental data are globally good, therefore new calculations do not appear as a priority. The PES adopted supports 47 vibrational levels, with a maximum of 236 rotational levels for $\mathrm{v}=0$. The calculated cross sections have as initial vibrational states $\mathrm{v}=0,1,3,5,7,10$, then 1 in 5 up to 35 , then all from 39 to 46 . Density of trajectories is limited to 4000 trajectories per $\mathrm{eV}$ per $\AA$, because the PES is much more complex, with the intermediate $\mathrm{O}_{3}$ which makes the average duration of each trajectory very long and also more difficult to integrate reliably with a large time step. As a result, two years of cpu has been dedicated to oxygen, while about one cpu year has been spent respectively for hydrogen and nitrogen, with a much higher trajectory density. Two different degeneracy factors have been applied to oxygen rate coefficients, one for vibrational energy exchange, temperature dependent [26], and another for dissociation, which is variable as a function of the initial rovibrational energy and has been proposed in [12].

\section{Cross section interpolation}
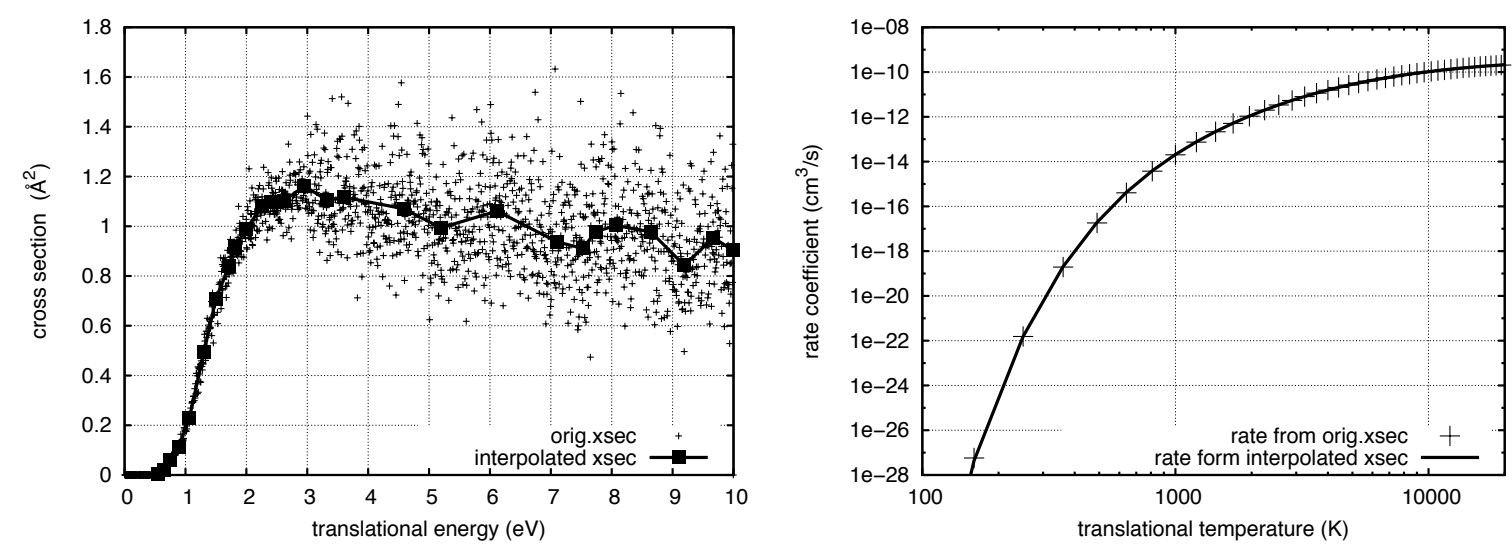

FIGURE 1. On the left: original cross section calculated subdividing energy axis into 1500 bins ("cloud"), in comparison with interpolating polygonal with not uniformly distributed points obtained as explained in the text. On the right: rate coefficient calculated from original cross section and from the interpolated one.

Interpolation of large sets of cross sections is, generally speaking, difficult for the presence of rapidly varying trends as a function of translational energy. To obtain computationally and then to reproduce these features with some kind of interpolation it is necessary to adopt particular care, because in some cases small errors in cross sections can bring easily to orders of magnitude of rate coefficient errors. The obvious starting point is to use a fine discretization of energy axis in cross section calculations, but this means to use large number of trajectories to populate the bins of the discretization, and then to accept a huge number of points to represent each cross section. This procedure is feasible from the molecular dynamics side, and in fact it has been applied for the three collisional systems presented here, with an energy interval of just $6 \mathrm{meV}$ and a "cloud" of points (bin values) to represent cross sections, but generally unacceptable from the point of view of a modelist, who has to include many different species and has to recall many times during a simulation an enormous amount of data. Interpolating these data is imperative, but the point to stress is that this interpolation should ideally shrink data retaining main features of each cross section, guaranteeing that rate coefficient is correctly reproduced using both originally detailed cross section and the 
interpolated one. Polynomial interpolation has been used during this work, but with not very reliable results, in particular the threshold region is generally very badly approximated, as well as any narrow peak. A refinement of this kind of interpolation requires a high order of polynomial with a relatively large number of coefficients (that is data to storage), but requires also a probably unacceptable high number of points to interpolate with small statistical errors, to avoid noisy polynomial oscillations. The technique proposed by the author consists of extracting the essential information of each "cloud" of cross section points with a method inspired by "feature extraction" typical of image processing. The idea is to find the symplest polygonal approximating the cross section cloud within a given tolerance. The polygonal has not a predetermined number of points, and the abscissas of its nodes is not predetermined at all: both these quantities are found by the algorithm with a progressive trial and error procedure, starting with just three points and then, if useful, increasing this number. This freedom gives an enormous advantage on more conventional schemes, because it allows to describe with sufficient accuracy both very slow trends as well as peaks, plateaus, thresholds, etc. with a very small number of parameters (that is two times the number of nodes of the polygonal). Numerical oscillations are simply non exisisting, and the method appears sufficiently robust to represent correctly both high value cross sections (with intrinsic high probability from the Monte Carlo point of view) as well as very rare events, with few non zero bin values. This robustness cannot be appreciated in only one figure, but it is very important in reproducing accurately the whole database. As a very limited example in fig. 1 on the left a typical cross section "cloud" is displayed with points, with its approximating polygonal. It is clear that in the first part of the cross section, up to $3 \mathrm{eV}$, there is a very fast increasing trend, while beyond that limit the trend is almost flat. As a consequence before $3 \mathrm{eV}$ the interpolation algorithm has placed about 16 points to follow accurately the rapidly varying curve, while in the remaining $7 \mathrm{eV}$ about other 13 points are used, to follow a relatively low detailed part of the cross section. This means a great advantage in having good global accuracy with an optimal number of points. To prove the accuracy of cross section reconstruction by means of polygonal, in fig. 1 on the right the rate coefficient obtained from original cross section and from interpolated one are compared, with good result on the whole range of temperatures. It is worthwhile to note that using the same number of points (about 30) uniformly distributed on energy axis it is quite difficult to obtain the same result, unless the trend is almost totally flat, but in that case the polygonal would be only made of three or four points.

The method, here and in [16] only sketched for what is interesting for its possible application in this context, is quite technical and will be presented with all the details in a specific publication.

\section{RATE COEFFICIENTS}

\section{Comparisons with literature}

It is important to assess the validity of calculations included into database, even if this is not simple due to the generally scarce presence of accurate, detailed and extended results in this field, in particular for vibrationaltranslational energy transfer. Some sparse data are available, often coming from deconvolution of averaged experimental quantities applying oversimplified models, which do not take into account multiquantum transitions, which in some systems can be quite important [13]. Simplified models for state-to-state rates have been recently compared with QCT calculations, showing a lacking of qualitative agreement $[27,28]$. The most reliable results are global thermal rate coefficients, and various good comparisons have been published elsewhere [6-16]. For the hydrogen collision process there is a complete database of computational origin available from Martin and Mandy, obtained with QCT method on LSTH PES. Some comparisons concerning vibration-translation energy exchange and dissociation significantly put in evidence the general agreement with data in this work obtained on a more accurate PES, but also some differences, particularly on high lying rovibrational states, which are determinant in recombination [29]. For nitrogen system, exchange rate has been compared with some global experimental and computational results, with quite good agreement $[8,20]$. Concerning oxygen, an interesting comparison can be appreciated with approximate experimental multiquantum transitions, showing qualitative agreement, with details described in [13]. However a much more detailed behaviour is described by calculations and not revealed by the simple experimental fit, which do not take into account final vibrational states. Dissociation has shown good agreement with thermal rates of experimental origin for the three collisional systems in this paper $[6,8,12]$, with good comparison also for oxygen if a particular variable multisurface factor proposed by the authors is applied. 


\section{Rotational transitions in vibrational monoquantum deactivation rates}

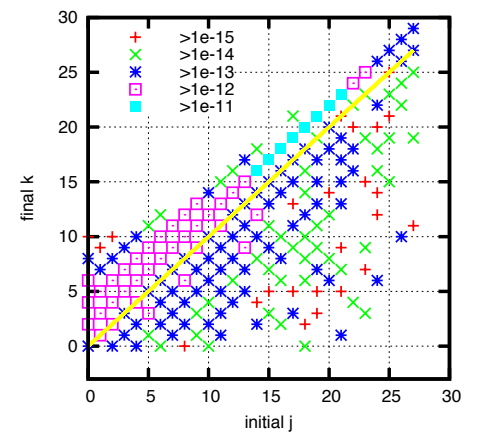

FIGURE 2. Rate coefficients for monoquantum vibrational non reactive deactivation from $\mathrm{v}=5$ for hydrogen with specified rovibrational initial and final rotational quantum numbers involved in the vibrational transition. Temperature is $300 \mathrm{~K}$. Rate coefficient values are subdivided in 5 orders of magnitude with the 5 symbols in the legend, starting from a minimum value of $10^{-15} \mathrm{~cm}^{3} / \mathrm{s}$.
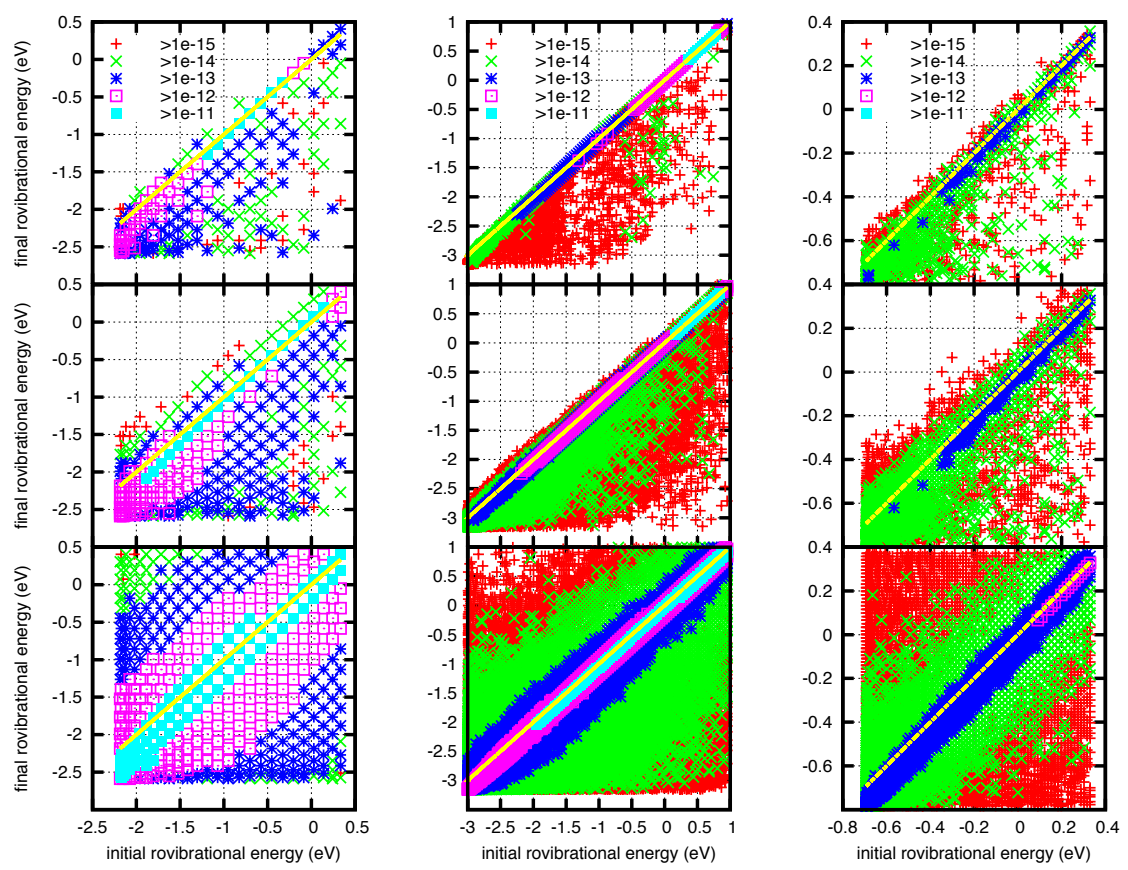

FIGURE.3. Rate coefficients for monoquantum vibrational non reactive deactivation from $v=5$ for hydrogen (first column), $v=30$ for nitrogen (second column) and oxygen (third column), with specified rovibrational energies of initial and final rotational states involved in the vibrational transition. In the first row temperature is $300 \mathrm{~K}$, in the second $1000 \mathrm{~K}$, in the third $10000 \mathrm{~K}$. Rate coefficient values are subdivided in 5 orders of magnitude as in the preceding figure.

In order to study the possibility of really introducing into models rotational dependence of rate coefficients (or cross sections) it is important to have an idea of the typical behaviour of this kind of rate coefficients when rotation is explicitly considered both for initial and final states, with particular attention to possible simplifications of these data when introduced into models. In fig. 2 a monoquantum vibrational deactivation transition is considered for hydrogen, in the non reactive case (no exchange of atoms), with initial $v=5$. On the abscissas all the initial rotational states $\mathrm{j}$ compatible with initial vibration are presented, while final rotational states $\mathrm{k}$ compatible with $\mathrm{w}=4$ are displayed on vertical axes. The values of the rate coefficient relative to a given couple $(\mathrm{j}, \mathrm{k})$ is represented by a red cross in a range from $10^{-15} \mathrm{~cm}^{3} / \mathrm{s}$ to $10^{-14} \mathrm{~cm}^{3} / \mathrm{s}$, with a green " $\mathrm{x}$ " from $10^{-14} \mathrm{~cm}^{3} / \mathrm{s}$ to $10^{-13} \mathrm{~cm}^{3} / \mathrm{s}$, and so on for 5 orders of magnitude, as illustrated in the legend. By following the accumulation of higher rates one can realize the distribution among rotational states of one "typical" vibrational rate ( $\mathrm{v}=5$ is an intermediate vibrational level for hydrogen). It is clear from the figure that the distribution of rate coefficients has an approximate diagonal symmetry, with the maximum density of highest rates on the upper subdiagonal, and many medium to low value rates on the 
other side of diagonal. This suggests that the rotational distribution is the highest for similar rotational quantum numbers $(\mathrm{j}, \mathrm{k})$, and more precisely for slightly higher exit $\mathrm{k}$ values than input $\mathrm{j}$ values. Considering the vibrational jump, one can suppose that the final rovibrational state with the nearest energy to that of initial one is the most probable. As a consequence of applying this hypothesis, rovibrational quantum numbers have been substituted by rovibrational energies in the two following figures. In fig. 3 the non reactive case is illustrated for hydrogen from $v=5$ (first column), nitrogen from $\mathrm{v}=30$ (second column), and oxygen from $\mathrm{v}=30$ (third column). In the first row temperature is $300 \mathrm{~K}$, in the second row is $1000 \mathrm{~K}$, in the third one $10000 \mathrm{~K}$. It is quite evident that the energetic criterium can explain easily the general diagonal trend common to all the graphs, in particular for medium to high rotational levels. For low lying rovibrational states there is a tendency to a final state less energetic than the initial one (the global vibrational transition is anyway exothermic), with some energy transferred to translation.
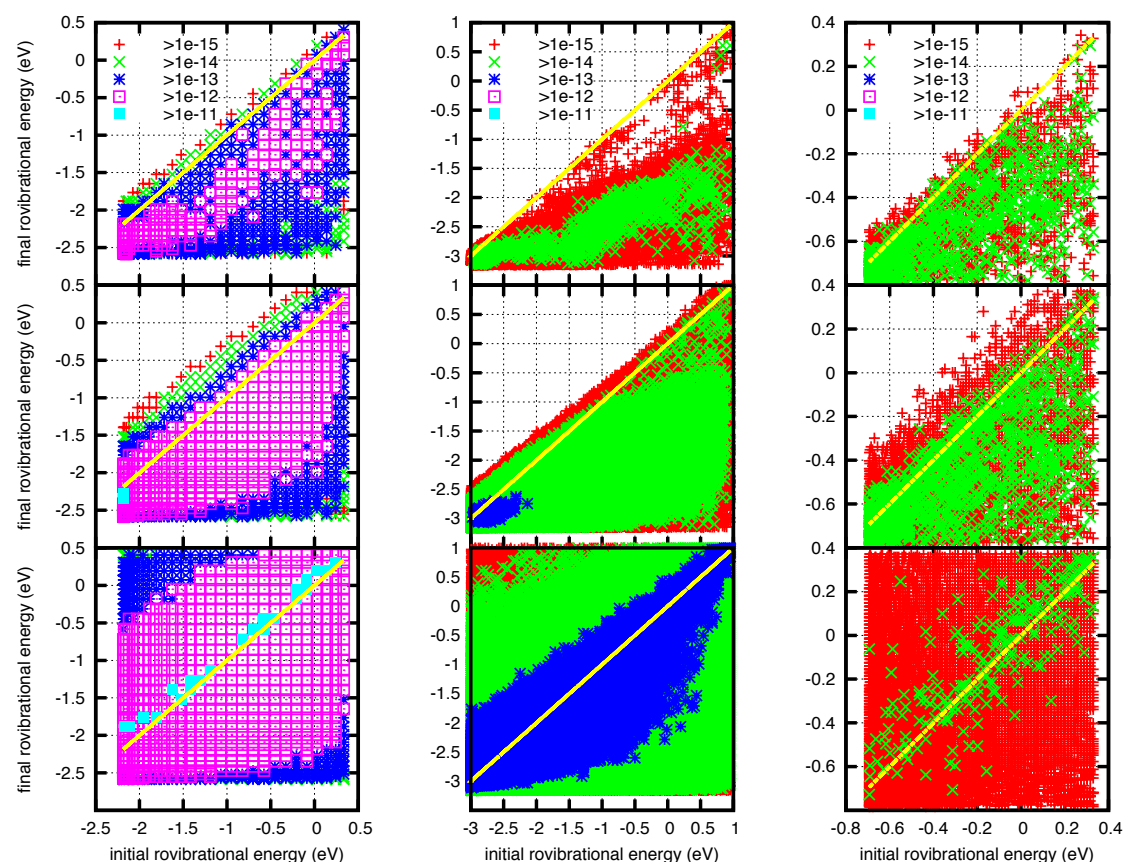

FIGURE 4. Rate coefficients for monoquantum vibrational reactive deactivation, symbols as in the preceding figure.

This effect appears, at least for the transitions taken into account, more important for hydrogen and oxygen, where many off-diagonal rates are within one order of magnitude from highest diagonal rates, and surely more important for high temperatures. For non reactive nitrogen treated with the LEPS PES, on the contrary, non diagonal terms appear lower than diagonal terms by two orders of magnitude or better, even at $\mathrm{T}=10000 \mathrm{~K}$. While up to $1000 \mathrm{~K}$ the upper subdiagonals are essentially unpopulated, for $\mathrm{T}=10000 \mathrm{~K}$ the distribution around the diagonal is almost perfectly symmetrical, with the exception of the low lying rotational states (roughly one third of the whole rotational ladder) which, as already underlined, tend to transfer some rovibrational energy to translation. The whole picture changes significantly, however, if reactive case is taken into account, as in fig.4, where all the other conditions are identical to the preceding figure. The energetic diagonal does not represent a locus of high rate coefficients for low to medium temperatures, on the contrary final rovibrational energy lower than initial one is a rule in this case, extended also to high rotational states, particularly for nitrogen and oxygen. At high temperature, the general trend is approximately symmetrical around the diagonal, but much more smoothed than in the non reactive case. The general behaviour is significantly different from the non reactive case, and unfortunately in a way that does not allow a simplification of rovibrational transitions, at least for the cases examined. An important exception is the nitrogen case, where the absolute values of reactive rates are anyway two orders of magnitude lower than the highest diagonal non reactive rates. It must be noted, however, that the nitrogen reaction has been treated here with semiempirical PES quite simple, therefore it is not unlikely that these findings might change using a more accurate PES. Concerning hydrogen, from the cases examined it is clear that a simplification of rovibrational distribution is impossible, because reactive rates are important and with a quite smooth and large distribution. In the oxygen case, there is a prevalence of diagonal non reactive rates, but only for one order of magnitude or less, therefore it is probably not safe to neglect reactive rates with their very diffuse distribution 


\section{Rotational states and dissociation/recombination dynamics}
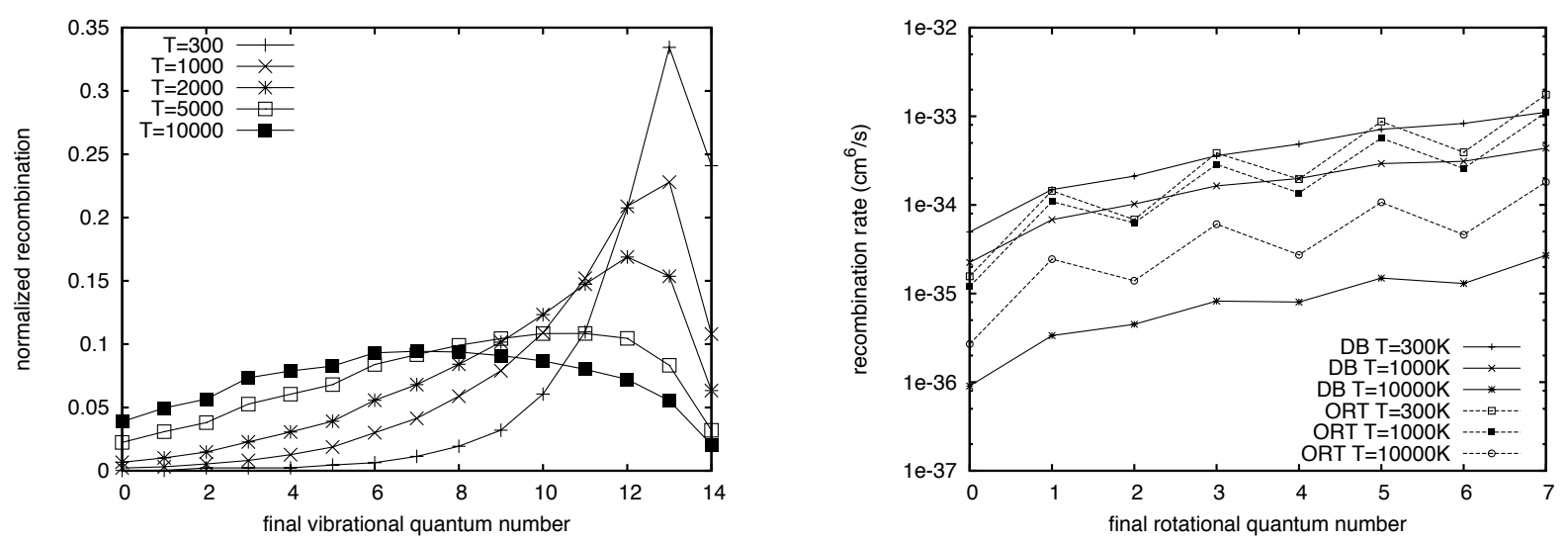

FIGURE 5. On the left: normalized hydrogen recombination as function of final vibrational quantum number at different temperatures from 300 to $10000 \mathrm{~K}$. On the right: hydrogen recombination for final vibrational quantum number $\mathrm{w}=13$, as a function of final rotational quantum number, at $\mathrm{T}=300,1000$ and $10000 \mathrm{~K}$, obtained with detailed balance (DB) and from quasibound kinetics (see text).

Concerning termolecular recombination: $A+B+C \rightarrow A+B C(v, j)$ this has been calculated essentially in two apparently very different ways. Following the first one, one calculates recombination from collision induced dissociation data obtained during QCT calculations applying detailed balance. It is important to underline that in this way recombination implicitly is considered a pure three body collision, that is the three bodies are supposed to enter interaction region almost at the same time. There is also another well known mechanism which explains and also gives the basis for calculating atomic recombination, the orbiting resonance theory (ORT [30]). In this case an equilibrium is supposed to exist between free atoms and orbiting resonances (quasibound states) of diatomic molecule, and the stabilization to bound states happens by collision with the third body. As illustrated in [31], there is a long history about the acceptance of ORT as the only method for determining recombination, even if some experimental facts are not explained satisfactorily within this theory. Smith [32] in a series of papers has clearly put the basis of a omnicomprehensive theory of termolecular recombination, in which each mechanism can really give its contribution. In [29] it has been shown that selecting accurately the states involved in the two mechanisms it is possibile to reconstruct a quite realistic recombination rate, with good comparisons with various heterogeneous data from literature. Really in that paper the authors have gone beyond the limits of ORT, including all the quasibound states, and introducing a temperature over pressure dependence of quasibound kinetics. Comparisons are good for specific results in literature, even if it has to be underlined the very large discrepancy (two to three orders of magnitude) between minimum and maximum recommended values for atomic recombination in hydrogen [33]. The only significantly different recombination result comes from a calculation of Krstic [34], with a dynamical method in which rotation is considered in an approximate way. It is interesting that in [29] limiting the number of rotational states included in recombination calculation one can find a result very similar to that of Krstic, as a further proof of the importance of rotation in dissociation/recombination dynamics. In fig.5 on the left hydrogen normalized recombination over final vibrational quantum number is shown at different values of temperature from 300 to $10000 \mathrm{~K}$, with a clear meaning: while at low temperature it is essentially correct to say that recombination takes place through high lying vibrational states, the distribution over vibrational ladder moves towards lower vibrational quantum numbers as temperature is raised, up to $10000 \mathrm{~K}$, when it is almost flat, with a slight prevalence of intermediate final vibrational states. Anyway, for any temperature, it is absolutely necessary to consider high lying rotational states, as evident in fig.5 on the right panel, where recombination rate coefficients calculated with detailed balance applied to dissociation (DB) and by ORT towards final vibrational quantum number $\mathrm{w}=13$ are presented as a function of final rotational quantum number for $\mathrm{T}=300,1000$ and $10000 \mathrm{~K}$. It is clear the increasing of recombination rates by orders of magnitude with final rotation, at any temperature and for both methods used.

\section{CONCLUSIONS}

Large databases of quasiclassical computed cross sections concerning atom-diatom collision processes for hydrogen nitrogen and oxygen have been presented, with motivations, methodology and comparisons with literature. 
A particular method for interpolation of large sets of cross sections has been proposed, with potentially interesting use into models. The importance of inclusion of rotational motion in a more or less detailed way into this kind of rate coefficients, with potential applications in models using those data, has been stressed. The distribution of rotational rate coefficients in a given vibrational transition has been studied for the three species, with different results. In particular, nitrogen rotational rates appear to be reducible with sufficient approximation not simply to their non reactive part, but to the subset having similar initial and final rovibrational energies. The large importance of rotational motion in dissociation/recombination dynamics has been presented with a study of hydrogen recombination including direct three body and quasibound kinetics mechanisms, with similar relevance in both cases.

\section{ACKNOWLEDGEMENTS}

This work has been supported by MIUR PRIN 2007 (2007H9S8SW 003)

\section{REFERENCES}

[1] G.Colonna, M.Tuttafesta, M.Capitelli, D.Giordano, Journal of Thermophysics and Heat Transfer 13, 372 (1999)

[2] M.Capitelli, G Colonna, F Esposito, Journal of Physical Chemistry A 108, 8930 (2004)

[3] G.Colonna, M.Capitelli, F.Esposito, AIAA-2003-3645

[4] G. Colonna, F.Esposito, M.Capitelli, AIAA-2006-3423

[5] G. Colonna, F. Esposito, M. Capitelli, AIP Conference Proceedings, 762, 1007 (2005)

[6] F.Esposito, C.Gorse, M.Capitelli, Chem. Phys. Lett. 303, 636-640 (1999)

[7] F.Esposito, M.Capitelli, Atomic and Plasma-Material Interaction Data for Fusion 9, 65-73 (2001).

[8] F.Esposito, M.Capitelli, Chem.Phys.Lett. 302, 49-54 (1999)

[9] F.Esposito, M.Capitelli, C.Gorse, Chem.Phys. 257, 193-202 (2000)

[10] F.Esposito, M.Capitelli, Chem. Phys. Lett. 418, 581-585 (2006)

[11] F.Esposito, I.Armenise, M.Capitelli, Chem. Phys. 331, 1-8,(2006)

[12] F.Esposito, M.Capitelli, Chem.Phys.Lett. 364, 180-187 (2002)

[13] F.Esposito, M.Capitelli, Chemical Physics Letters 443, 222-226 (2007)

[14] F.Esposito, I.Armenise, G.Capitta, and M.Capitelli, Chem. Phys. 351, 91-98 (2008)

[15] F.Esposito, M.Capitelli, AIP Conference Proceedings 771, 246 (2005)

[16] F.Esposito, in Non equilibrium phenomena plasma combustion atmosphere, NEPCAP 2009, Sochi (Russia), Ed. G.D.Roy, S.M.Frolov, A.M.Starik, Torus Press, Moscow 2009, pp.3-11

[17] F.Esposito, Ph.D Thesis, University of Bari, Italy (1999).

[18] Boothroyd, A. I., Keogh, W. J., Martin, P. G., Peterson, M. R., J. Chem. Phys. 104, 7139 (1996)

[19] Lagana', A., Garcia, E., and Ciccarelli, L., J. Phys. Chem. 91, 312 (1987)

[20] D. Wang, J. R. Stallcop, W. M. Huo, C. E. Dateo, D. W. Schwenke, and H. Partridge, JCP 118, 2186 (2003)

[21] E. Garcia, A. Saracibar, S. Gomez-Carrasco and A. Lagana, Phys. Chem. Chem. Phys., 10, 2552 (2008).

[22] B.L. Galvao and A.J.C. Varandas, J. Phys. Chem. A 113,14424 (2009)

[23] A.J.C. Varandas, A.A.C.C. Pais, Mol. Phys. 65, 843 (1988).

[24] R. Siebert, R. Schinke, M. Bittererova, Phys. Chem. Chem. Phys. 3 , 1795 (2001).

[25] D. Babikov, B.K. Kendrick, R.B. Walker, R.T. Pack, P. Fleurat- Lessard, R. Schinke, J. Chem. Phys. 118, 6298 (2003).

[26] A. Gross, G.D. Billing, Chem. Phys. 217, 1 (1998).

[27] M.Ju. Pogosbekian, A.L. Sergievskaia, and S.A. Losev, Chemical Physics, 328, 371-378 (2006)

[28] F.Esposito, M.Lino da Silva, "Comparison of VT rate coefficients for $\mathrm{N}^{+} \mathrm{N}_{2}(\mathrm{v})$ calculated by quasiclassical method and by forced harmonic oscillator method", unpublished work

[29] F. Esposito, M. Capitelli, J. Phys. Chem. A, 113, 15307-15314 (2009)

[30] R. E. Roberts, R. B. Bernstein, C. F. J. Curtiss, Chem. Phys. 50, 5163 (1969).

[31] R. T. Pack, R. B. Walker, B. K. Kendrick, J. Chem. Phys. 109, 6701 (1998).

[32] F.Smith, "Chemical Reactions in High-Temperature Gases as Collision Processes" in Kinetic Processes in Gases and Plasmas, edited by A. R. Hochstim, New York: Academic, 1969, pp 257-280.

[33] K. K. Wetzel, C. J. W. Solomon, Propul. Power 10, 492 (1994).

[34] P. S. Krstic, R. K. Janevand, D. R. Schultz, J. Phys. B: At. Mol. Opt. Phys., 36, L249-L255 (2003). 